OPEN ACCESS

Edited by:

Peter Schröder,

German Research Center for Environmental Health (HZ),

Germany

Reviewed by:

Uener Kolukisaoglu,

University of Tubingen, Germany

Jacqueline Vanni Shanks,

lowa State University, United States

*Correspondence:

Elizabeth L. Rylott

liz.rylott@york.ac.uk

Neil C. Bruce

neil.bruce@york.ac.uk

Specialty section:

This article was submitted to

Plant Physiology,

a section of the journal

Frontiers in Plant Science

Received: 14 August 2018 Accepted: 28 November 2018 Published: 12 December 2018

Citation:

Tzafestas K, Ahmad L, Dani MP, Grogan G, Rylott EL and Bruce NC (2018) Structure-Guided Mechanisms

Behind the Metabolism of 2,4,6-Trinitrotoluene by Glutathione Transferases U25 and U24 That Lead

to Alternate Product Distribution.

Front. Plant Sci. 9:1846.

doi: 10.3389/fpls.2018.01846

\section{Structure-Guided Mechanisms Behind the Metabolism of 2,4,6-Trinitrotoluene by Glutathione Transferases U25 and U24 That Lead to Alternate Product Distribution}

\author{
Kyriakos Tzafestas', Laziana Ahmad',2, M. Paulina Dani', Gideon Grogan², \\ Elizabeth L. Rylott ${ }^{1 *}$ and Neil C. Bruce ${ }^{1 *}$ \\ ${ }^{1}$ Centre for Novel Agricultural Products, Department of Biology, University of York, York, United Kingdom, ${ }^{2}$ York Structural \\ Biology Laboratory, Department of Chemistry, University of York, York, United Kingdom
}

The explosive xenobiotic 2,4,6-trinitrotoluene (TNT) is a major worldwide environmental pollutant and its persistence in the environment presents health and environmental concerns. The chemical structure of TNT dictates that biological detoxification pathways follow predominantly reductive transformation of the nitro groups, and as a result, TNT is notoriously recalcitrant to mineralization in the environment. Plant-based technologies to remediate this toxic pollutant rely on a solid understanding of the biochemical detoxification pathways involved. Toward this, two Arabidopsis Tau class glutathione transferases, GSTU24 and GSTU25, have been identified that catalyze the formation of three TNT-glutathionylated conjugates. These two GSTs share $79 \%$ identity yet only GSTU25 catalyzes the substitution of a nitro group for sulfur to form 2-glutathionyl-4,6dinitrotoluene. The production of this compound is of interest because substitution of a nitro group could lead to destabilization of the aromatic ring, enabling subsequent biodegradation. To identify target amino acids within GSTU25 that might be involved in the formation of 2-glutathionyl-4,6-dinitrotoluene, the structure for GSTU25 was determined, in complex with oxidized glutathione, and used to inform site-directed mutagenesis studies. Replacement of five amino acids in GSTU24 established a conjugate profile and activity similar to that found in GSTU25. These findings contribute to the development of plant-based remediation strategies for the detoxification of TNT in the environment.

Keywords: 2,4,6-trinitrotoluene, TNT, Arabidopsis, glutathione transferase, GST, detoxification, xenobiotic

\section{INTRODUCTION}

The continual use of explosives, along with production and decommissioning is progressively contaminating military sites worldwide (Amaral et al., 2009; Zheng et al., 2009). The total area of operational ranges in the United States contaminated with munitions constituents is estimated to be more than 10 million hectares (United States General Accounting Office, 2004). Pollution in European countries, from former WWII manufacturing and disposal sites is also widespread 
(Spain et al., 2000). The most broadly used explosive, 2,4,6trinitrotoluene (TNT) is associated with extensive soil and water contamination (Lewis et al., 2004). Contaminated training ranges have hotspots of TNT that can reach concentrations of up to $87000 \mathrm{mg} \mathrm{kg}^{-1}$ soil (Talmage et al., 1999), with 100-1000 mg $\mathrm{kg}^{-1}$, or lower for surface soils in artillery training ranges and 1-36 mg kg-1 for hand grenade ranges (Jenkins et al., 2006; Clark and Boopathy, 2007).

Nitro-substituted organic compounds, such as TNT, pose a specific challenge to plant and bacterial degradation. The electron withdrawing nitro groups on the TNT molecule provide stability to the aromatic ring through resonance, rendering the ring particularly resistant to oxidative attack and subsequent ring cleavage (Qasim et al., 2009). Thus TNT is particularly recalcitrant to biodegradation and persists in the environment (Rylott et al., 2011).

In a previous study, two Arabidopsis thaliana (Arabidopsis) glutathione transferases, GSTU24 and GSTU25, were shown to conjugate TNT to glutathione (GSH) producing three distinct TNT-GSH conjugates, shown in Figure 1 (Gunning et al., 2014). For two of the compounds, GSH conjugation occurred through the methyl group of TNT; however, the third conjugate (conjugate 3 ) resulted from the nucleophilic substitution of a nitro group to form 2-glutathionyl-4,6-dinitrotoluene (GDNT). Replacement of the nitro group with sulfur could destabilize the aromatic ring. Fungi and bacteria with the ability to mineralize dinitrotoluenes exist (Valli et al., 1992; Nishino et al., 2000; Johnson et al., 2002) and enzymatic pathways for DNT biodegradation have been characterized (Nishino et al., 2000; Johnson et al., 2002). Thus, production of GDNT could present an opportunity for cleavage and subsequent biodegradation of this toxic environmental pollutant.

Plant GSTs are a superfamily of enzymes: In Arabidopsis, there are 54 GSTs subdivided into seven classes. While many GSTs are able to conjugate GSH to a wide range of xenobiotic substrates, they are also involved in catalyzing ascorbate recycling and various metabolic reactions, with some GSTs also exhibiting glutathione peroxidase (GPOX) activity (Dixon and Edwards, 2010), and non-enzymatic ligand binding properties (Smith et al., 2003; Dixon et al., 2011). The Tau class, to which GSTU24 and GSTU25 belong, can be subdivided into three distinct clades. Many of the GSTs within the clade GSTU19 to GSTU28 are implicated in the detoxification of xenobiotics such as herbicides and safeners (Edwards et al., 2005; Labrou et al., 2015). Expression of both GSTU24 and GSTU25 is induced by TNT, with GSTU25 also exhibiting relatively high GPOX activity (Dixon and Edwards, 2009) and activity toward the model substrate 1-chloro-2,4-dinitrobenzene (CDNB; Mezzari et al., 2005; Gandia-Herrero et al., 2008). To date, Tau class GSTs are unique in their ability to bind glutathione-conjugated fatty acid derivatives (Mezzari et al., 2005; Dixon and Edwards, 2009), with GSTU25 known to selectively bind hydroxylated fatty acids. Yet, despite the mounting knowledge on these enzymes, the endogenous roles for GSTU24 and GSTU25, and the vast majority of plant GSTs in general, remains elusive.

The structures of several Tau class plant GSTs have been solved: The wheat (Triticum aestivum), TaGSTU4-4 structure was determined in complex with $S$-hexylglutathione (Thom et al., 2002) and rice (Oryza sativa) OsGSTU1 (Protein Data Bank code 1OYJ), while two soybean (Glycine max) GSTs have been determined; GmGST-U4-4 in complex with $S$-( $p$-nitrobenzyl)glutathione (Axarli et al., 2009) and GmGSTU10-10 (Skopelitou et al., 2015). Although there is high protein sequence variability between these GSTs, the structures are remarkably conserved (Dixon and Edwards, 2010; Skopelitou et al., 2015). Existing as soluble homo or heterodimers, each $23-30 \mathrm{kDa}$ subunit is 200-300 amino acids in length. Within each subunit is a kinetically independent active site containing $\mathrm{G}$ and $\mathrm{H}$ sites. The $\mathrm{G}$ site, which is relatively well conserved, is formed from the $\mathrm{N}$-terminal domain which exhibits $\alpha / \beta$ topology, and binds GSH and, less commonly, other closely related peptides. The H-site exists within an $\alpha$-helical structure in the C-terminal domain but is less well conserved than the G site and, as a result, GSTs have wide substrate specificity.

Only $1.3 \mathrm{~kb}$ apart on chromosome I, GSTU24 and GSTU25 share $79 \%$ protein identity, indicative of a relatively recent gene duplication event. In this study, we report the structure of GSTU25. We then use this structure, in combination with alignment from other Tau-class plant GSTS whose structures have previously been solved (Axarli et al., 2009, 2016), to predict the key amino acids in the active site of GSTU25 that are associated with the specificity of the conjugation reactions of TNT with GSH.

\section{MATERIALS AND METHODS}

\section{Expression and Protein Purification for Crystallization}

The GSTU24 and GSTU25 from A. thaliana (Arabidopsis) ecotype Col0, and mutants, were cloned from pET-YSBLIC3C (described below) into pET22a to remove the his-tag, then transformed into Escherichia coli Tuner (DE3) cells (Novagen) that also contained the pRARE plasmid from Rosetta (Novagen). Transformants were grown on agar plates of Luria Bertani medium containing kanamycin $\left(100 \mu \mathrm{g} \mathrm{mL}^{-1}\right)$ and $50 \mu \mathrm{g} \mathrm{mL}^{-1}$ chloramphenicol $\left(50 \mu \mathrm{g} \mathrm{mL}^{-1}\right)(\mathrm{LB}+\mathrm{KC})$. A single colony of a plate grown overnight was used to inoculate a $5 \mathrm{~mL}$ starter culture of $\mathrm{LB}+\mathrm{KC}$ medium, which was grown overnight at $37^{\circ} \mathrm{C}$, $180 \mathrm{rpm}$. The starter culture was then used to inoculate $400 \mathrm{~mL}$ $\mathrm{LB}+\mathrm{KC}$ medium which was incubated at $37^{\circ} \mathrm{C}$ with shaking until an $\mathrm{OD}_{600}$ of $0.5-0.8$ was reached. At this point expression of the GST was induced by the addition of isopropyl $\beta-\mathrm{D}-1$ thiogalactopyranoside (IPTG, final concentration of $1 \mathrm{mM}$ ) and culture incubated at $20^{\circ} \mathrm{C}, 180 \mathrm{rpm}$. After approximately $18 \mathrm{~h}$ growth, cells were harvested by centrifugation at $5000 \mathrm{~g}$ for $15 \mathrm{~min}$ then resuspended in $20 \mathrm{mM}$ Tris/ $\mathrm{HCl}$ buffer $\mathrm{pH}$ 7.5. Cells were disrupted by ultrasonication, centrifuged at $15,000 \mathrm{~g}$ for $30 \mathrm{~min}$ then the supernatant loaded onto a $10 \mathrm{~mL} \mathrm{GSH}$ Sepharose 4B (GE healthcare). Column fractions were analyzed by SDS-PAGE and the fraction containing purified proteins were pooled and concentrated using a $10 \mathrm{kDa}$ cut-off Centricon ${ }^{\circledR}$ filter membrane. Concentrated protein was loaded onto an S75 Superdex ${ }^{\mathrm{TM}}$ gel filtration column and fractions containing pure 

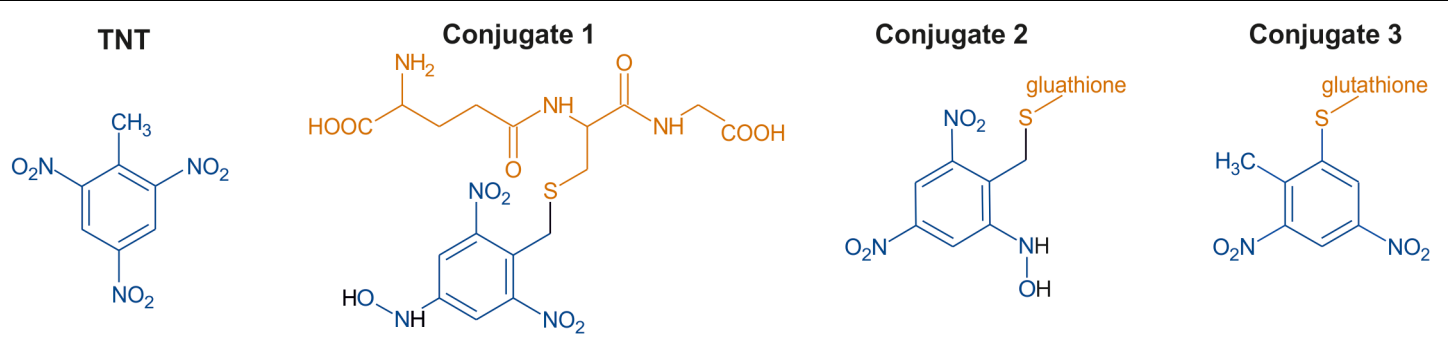

FIGURE 1 | Chemical structures of 2,4,6-trinitrotoluene (TNT) and the three glutathione-TNT conjugates, as determined by Gunning et al. (2014).

protein, as determined by SDS-PAGE, were pooled and stored at $-20^{\circ} \mathrm{C}$.

\section{Protein Crystallization}

Commercially available crystallization screens in 96-well plate sitting drop format were pre-incubated with $2 \mathrm{mM} \mathrm{GSH}$ and $2 \mathrm{mM}$ TNT in $54 \mu \mathrm{L}$ of reservoir solution in reservoir well. Pure AtGSTU25 was then subjected to crystallization trials using a Mosquito ${ }^{\circledR}$ ROBOT (TTP LabTech) in which each drop contained $150 \mathrm{~nL}$ protein and $150 \mathrm{~nL}$ precipitant reservoir solution. Initial crystals observed for the complex of AtGSTU25 mixture were obtained in solutions containing $0.2 \mathrm{M}$ ammonium acetate, $0.1 \mathrm{M}$ bis-tris propane pH 5.5 and 25\% (w/v) PEG 3350. Larger crystals for diffraction analysis were obtained using the hanging-drop vapor diffusion method in 24-well plate Linbro dishes with $2 \mu \mathrm{L}$ drops of a ratio of mother liquor to protein solution. The best crystals of the complex of GSTU25 with oxidized glutathione were obtained in crystal drops containing $0.2 \mathrm{M}$ ammonium acetate, $0.1 \mathrm{M}$ bis-tris propane $\mathrm{pH} 5.5$ and 23\% (w/v) PEG 3350. Prior to analysis on in-house X-ray equipment, the crystals were washed with the reservoir solution containing $20 \%$ (v/v) ethylene glycol as the cryoprotectant, followed by flashcooling in the liquid nitrogen. Crystals were tested for diffraction using a Rigaku Micromax-007HF fitted with Osmic multilayer optics and a MARRESEARCH MAR345 imaging plate detector. Those crystals that diffracted to a resolution of equal to, or better than, $3 \AA$ were retained for dataset collection at the synchrotron.

\section{Data Collection, Structure Solution, Model Building, and Refinement}

The complete dataset described in this report was collected at the Diamond Light Source, Didcot, United Kingdom on beamline I02. The data were processed and integrated using XDS (Kabsch, 2010) and scaled using SCALA (Evans, 2006) included in the Xia2 processing system (Winter, 2010). Data collection statistics are provided in Table 1. All crystals of U25 were obtained in space group $P 2{ }_{1} 22_{1}$, with four molecules in the asymmetric unit, constituting two dimers. The structure of AtGSTU25 was solved using MOLREP (Vagin and Teplyakov, 1997), with a monomer of the structure of the Tau class glutathione transferase from G. max (PDB 4TOP; 65\% sequence identity) as a model. The solvent content in crystals was $51 \%$. Structures were built and refined using iterative cycles using Coot (Emsley and Cowtan, 2004) and REFMAC (Murshudov et al., 1997), employing local NCS restraints in the refinement cycles. After building and refinement of the protein and water molecules, clear residual density was observed in the omit maps at the GSH binding site. This could be clearly modeled as glutathione disulfide (GSSG). The final structure exhibited $R_{\text {cryst }}$ and $R_{\text {free }}$ values of 20.5 and $21.7 \%$, respectively. All structures were validated and checked using PDB validation software upon deposition. Refinement statistics for all structures are presented in Table 1. The Ramachandran plot for AtGSTU25-GSSG showed $98.4 \%$ of residues to be situated in the most favored regions, $1.1 \%$ in additional allowed and $0.5 \%$ residues in outlier regions.

\section{Generation of the GST Mutants}

A QuickChange II Site-Directed Mutagenesis Kit (Agilent Technologies) was used to generate the mutants, using the primers listed in Table 2. Wild-type and mutated GSTU24 and GSTU25 were cloned into pET-YSBLIC3C, used to transform E. coli (BL21) cells, and expressed and purified as described previously (Gunning et al., 2014).

TABLE 1 | Data collection and refinement statistics for GSTU25-GSSG complex.

\begin{tabular}{|c|c|}
\hline Beamline & Diamond 102 \\
\hline Wavelength $(\AA ̊)$ & 0.97949 \\
\hline Resolution $(\AA ̊)$ & $48.54-1.95(1.99-1.95)$ \\
\hline Space group & $P 2{ }_{1}{ }_{1}{ }_{2}{ }_{1}$ \\
\hline Unit cell (Å) & $\begin{array}{c}a=87.83 ; b=107.67 ; c=108.75 \\
\alpha=\beta=\gamma=90^{\circ}\end{array}$ \\
\hline No. of molecules in the asymmetric unit & 4 \\
\hline Unique reflections & $75638(4446)$ \\
\hline Completeness (\%) & $99.8(100.0)$ \\
\hline$R_{\text {merge }}(\%)$ & $0.07(0.54)$ \\
\hline$R_{\text {p.i.m. }}$ & $0.05(0.36)$ \\
\hline Multiplicity & $6.4(6.2)$ \\
\hline$<1 / \sigma(l)>$ & $12.7(3.0)$ \\
\hline Overall $B$ factor from Wilson plot $\left(\AA^{2}\right)$ & 25 \\
\hline$R_{\text {cryst }} / R_{\text {free }}(\%)$ & $20.5 / 21.7$ \\
\hline r.m.s.d $1-2$ bonds $(\AA)$ & 0.02 \\
\hline r.m.s.d $1-3$ angles $\left({ }^{\circ}\right)$ & 1.94 \\
\hline Avge main chain $B\left(\AA^{2}\right)$ & 31 \\
\hline Avge side chain $B\left(\AA^{2}\right)$ & 35 \\
\hline Avge water $B\left(\AA^{2}\right)$ & 42 \\
\hline Avge ligand $B\left(\AA^{2}\right)$ & 40 \\
\hline
\end{tabular}

Numbers in brackets refer to data for highest resolution shells. 
TABLE 2 | Primers used for the site-directed mutagenesis of GSTU24 and GSTU25.

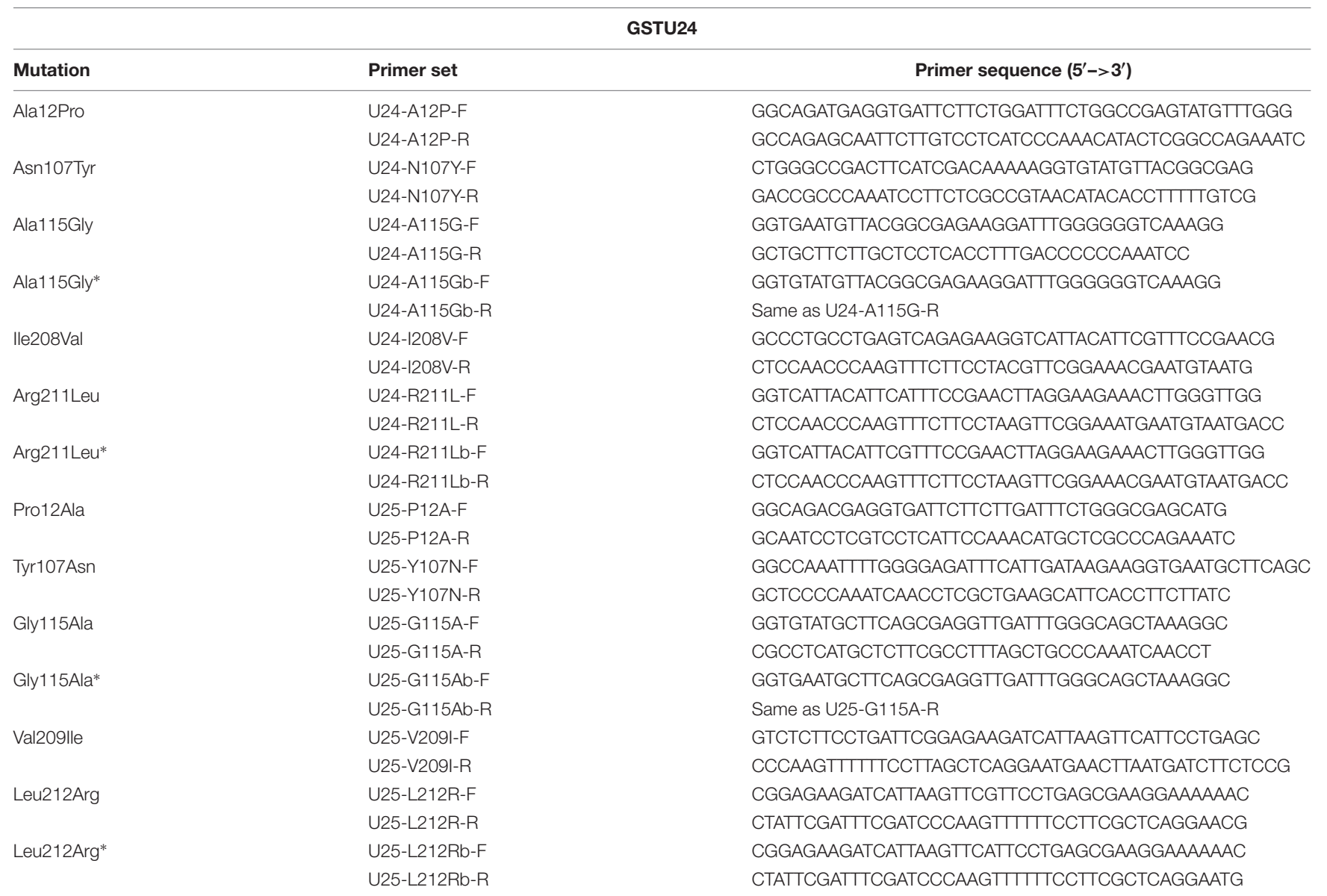

The asterisks (*) mark primer sets that were designed for the generation of sequential mutations and carry in their sequence the previous mutation, e.g., the Ala115Gly* primer set is designed to insert the Ala115Gly mutation into a sequence that already has the Asn107Tyr mutation.

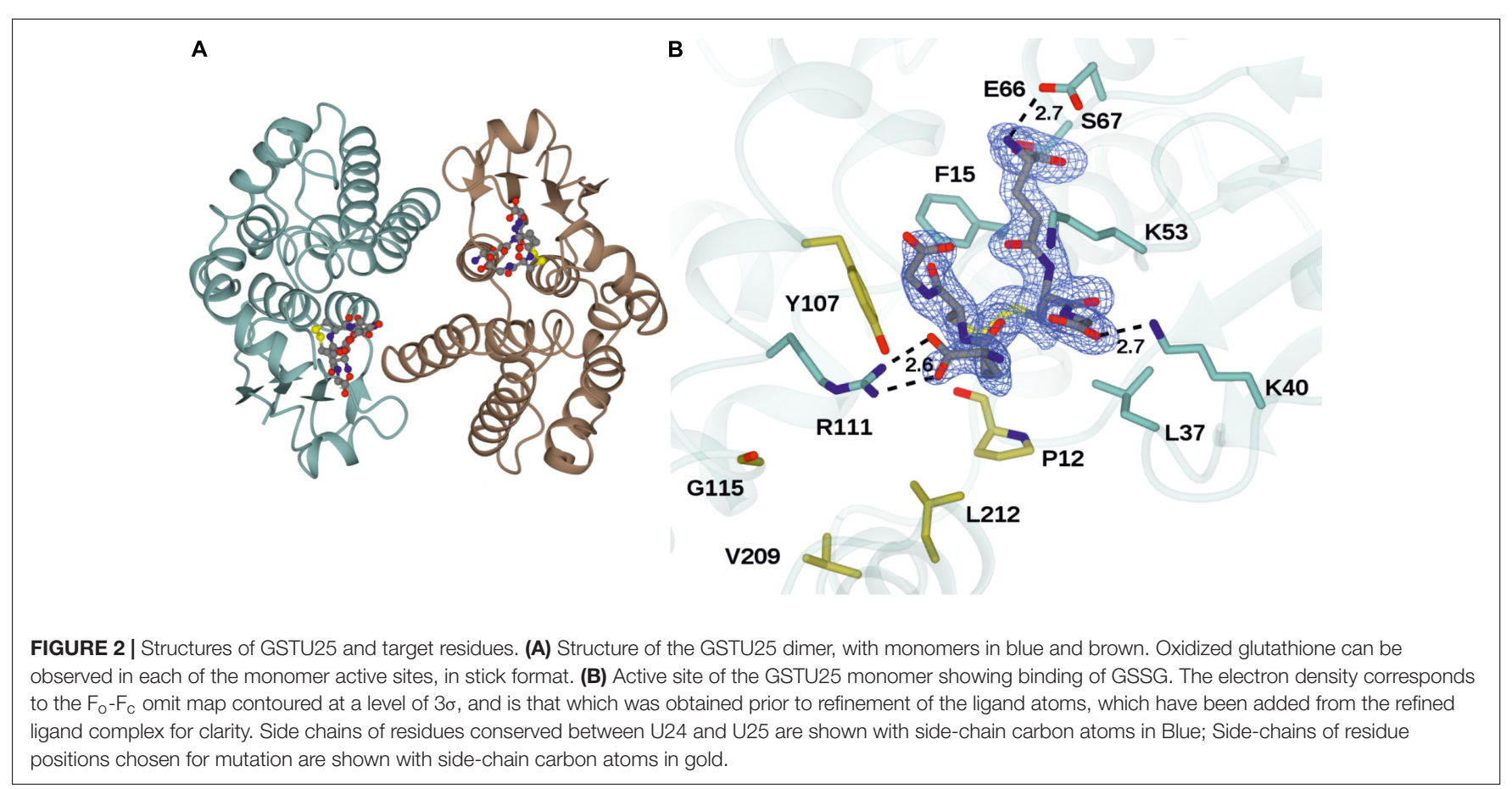




\section{GST Assays Using CDNB}

Conjugating activity of the purified proteins, and crude extracts from rosette leaves, was assessed using $\mathrm{CDNB}$ as described previously (Colville and Smirnoff, 2008). Briefly, the reaction, at $25^{\circ} \mathrm{C}$, comprised $100 \mathrm{mM}$ potassium phosphate buffer $\mathrm{pH}$ $6.5,5 \mathrm{mM} \mathrm{GSH}$ and a range of CDNB concentrations, and was initiated by addition of $5 \mu \mathrm{g}$ of purified enzyme to a final volume of $1 \mathrm{~mL}$. Increase in absorbance at $A_{340}$ was measured spectrophotometrically. The Michaelis-Menten plots and kinetic calculations $\left(\mathrm{K}_{\mathrm{m}}\right.$ and $\left.\mathrm{V}_{\max }\right)$ were performed using SigmaPlot 14 software. Statistical analysis was performed using Statistical Package for Social Sciences (SPSS) software (version 25, SPSS, Inc., Chicago, IL, United States). Results were analyzed using the analysis of variance (ANOVA) for continuous variables. $P$-values $<0.05$ were considered to be statistically significant.

\section{GST Assays Using TNT}

Reactions, carried out at $20^{\circ} \mathrm{C}$, contained $100 \mathrm{mM}$ potassium phosphate buffer $\mathrm{pH} 7.0,300 \mu \mathrm{g}$ of purified enzyme and $5 \mathrm{mM}$ GSH and was initiated by addition of TNT to a final volume of $250 \mu \mathrm{L}$. Reactions were stopped by the addition of trichloroacetic acid, to a final concentration of $10 \%(\mathrm{v} / \mathrm{v})$, and samples analyzed by HPLC.

\section{Measurement of TNT and Products}

The TNT and conjugates were analyzed by HPLC using a Waters HPLC system (Waters 2695 separator and Waters Photodiode array detector) with Waters X-Bridge C18 column $(300 \mathrm{~mm} \times 4.5 \mathrm{~mm}, 5 \mu \mathrm{M})$. The mobile phases for the gradient conditions were: mobile phase A, acetonitrile; mobile phase $\mathrm{B}$, $50 \mathrm{mM} \mathrm{NaH}_{2} \mathrm{PO}_{4}, \mathrm{pH} 2.7$, with $85 \%$ (v/v) phosphoric acid. The gradient ran: 0 min 0\% A 100\% B, 6 min 0\% A 100\% B, 11 min $50 \%$ A $50 \%$ B, $25 \min 100 \%$ A $0 \%$ B, $30 \min 0 \%$ A $100 \%$ B, runtime $30 \mathrm{~min}$. Integration was performed at $250 \mathrm{~nm}$ with Empower Pro Software.

\section{Nitrite Measurement}

Nitrite production was assayed according to the method of French et al. (1998) with modifications as described in Gunning et al. (2014).

\section{Probing the Mutants With ANS}

The ANS binding assay, based on the protocol by Yang et al. (2009), was used to determine conformational changes. The assay was performed in a $1 \mathrm{~mL}$ cuvette with $100 \mu \mathrm{l}$ of $2 \mathrm{mM}$ ANS, $50 \mu \mathrm{g}$ of enzyme and $100 \mathrm{mM}$ potassium phosphate buffer $\mathrm{pH} 6.5$. The fluorescence emission was monitored using a FluoroMax ${ }^{\circledR}-4$ Spectrofluorometer (Horiba Scientific).

\section{Accession Numbers}

AtGSTU24; TAIR accession number Atlg17180, AtGSTU25; TAIR acc. no. At1g17170. AtGSTU25-GSSG coordinates; Protein Databank (PDB) acc. no. 5g5a. GmGSTU4-4; PDB acc. no. 2VO4, Sh14; PDB acc. no. 5AGY, PcUre2pA; PDB acc. no. 4F0B, EcYghU; PDB acc. no. 3C8E, EcYfcG; PDB acc. no. 3GX0, CoGRX2; PDB acc. no. 4TR0.

\section{RESULTS}

\section{Structure of GSTU25}

The structure of GSTU25 was solved using molecular replacement at a resolution of $1.99 \AA$ with GmGSTU4-4 as template (Axarli et al., 2009). Analysis of the protein structure using the DALI server (Holm and Rosenstrom, 2010) revealed that the monomer was more similar to the structure of a Tau class GST mutant from G. max, called Sh14 (Axarli et al., 2016). Both structures were $68 \%$ identical, with a RMS value of $1.2 \AA$ over 219 residues. Each monomer of GSTU25 has four $\beta$-strands and nine $\alpha$-helices adopting the canonical GST fold. The first 77 residues at the $\mathrm{N}$-terminus fold into a thioredoxin-like domain followed by an $\alpha$-helical domain at the C-terminus from position 89 to 216 , with the two domains connected together by a short linker. Although the crystals were incubated with TNT and GSH, binding of TNT was not detected. Instead, multiple rounds of structure refinement cycles using the REFMAC5 program (Murshudov et al., 2011) revealed two GSH molecules covalently linked by a disulfide bond, showing the structure of GSTU25 in complex with glutathione disulfide (GSSG) (Figure 2A). At the binding site, the GSSG subunits: GSH-1 and GSH-2, were located in a binding pocket surrounded by polar, non-polar and charged amino acids (Figure 2B). This pocket was similar to the

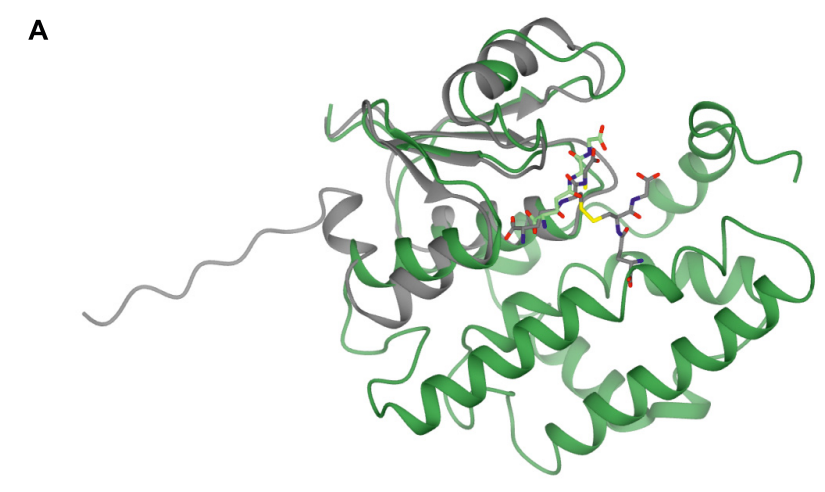

B

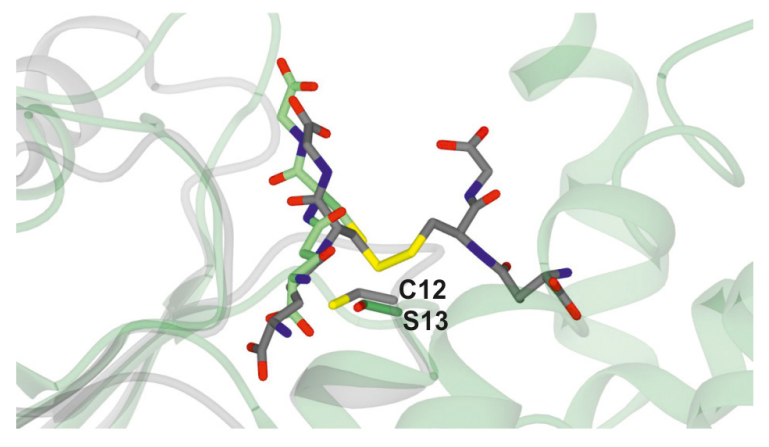

FIGURE 3 | Comparison of GSTU25 with CoGRX2. (A) Superimposed structures of the glutaredoxin subunit from Clostridium oremlandii (CoGRX2 in complex with GSSG (C-atoms in gray), and the GSTU25 subunit (green) in complex with GSSG (C-atoms in green). The RMS value for the superimposed structures is $2.3 \AA$ over 73 residues. (B) Position of the active residue for GSH thiol stabilization: serine 13, in GSTU25 and cysteine 12 in CoGRX2. 


\section{TaGSTU4-4 \\ OSGSTU1 \\ GmGSTU10-10 \\ GmGSTU4-4 \\ AtGSTU2 4 \\ AtGSTU25}

TaGSTU4-4

OSGSTU1

GmGSTU10-10

GmGSTU4-4

AtGSTU2 4

AtgSTU25

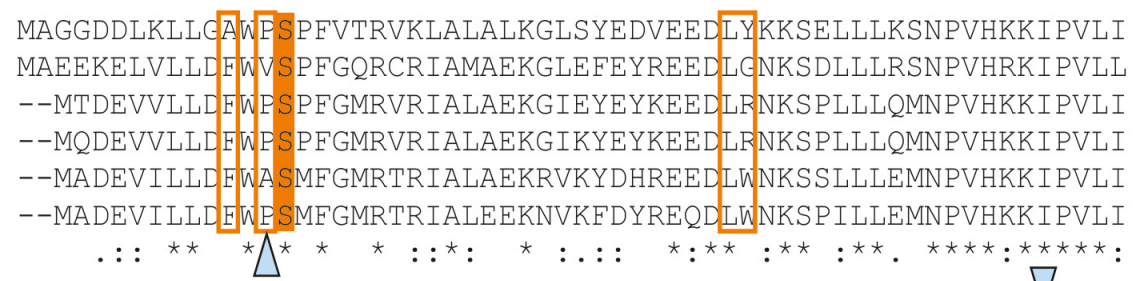

MKVTEALSGDKIFDAAKTPLLAAWVERFIELDAAKAALPDVGRLLEFAKA RAAAAASKFYSYERCGGFSV--EEVAPRLAAWARRCGRIDSVVKHLPS PEKVYDFVGVIIKKKYGVE-FKVYETFGSLNI--ENECPRFVAWAKRCLQKESVAKSLPDQHKVYEFV VEIRKKLVIEFKAYETFGTLNI--ESECPKEIAWAKRCLQKESVAKSLPDQQKVYEFIMDIRKKLGIE-FAVYEKFGNVS I --ESECSKLVAWAKRCLERESVAKALPESEKVITFISERRKKLGLE-FEAYEKFGSFS I--EAECPKLIAWGKRCVERESVAKSLPDSEKIIK|FV]PEIRKKLGIEIE

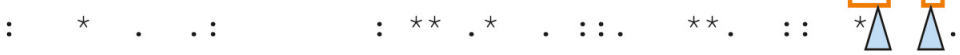

\begin{tabular}{|c|c|c|}
\hline * & Indentity conserved & Residues important to substrate specificity \\
\hline : & Similarity conserved & Residues forming the hydrophobic $\mathrm{H}$-site \\
\hline & Ser13 catalytic residue & Residues targeted for reciprocal site-directed mutagenesis in U24 and U25 \\
\hline
\end{tabular}

FIGURE 4 | Multiple sequence alignment of Tau class GSTs. Figure generated using Clustal Omega (Sievers et al., 2011).

active site identified for most GSTs, where the hydroxyl group of S13 and Y107 has been shown to contribute to the ionization of the GSH sulfhydryl group (Brock et al., 2013). Similar locations were observed for the same $\mathrm{S}$ and Y residues of GmGSTU4-4 in complex with $S$-(p-nitrobenzyl)-glutathione (Axarli et al., 2009). The S residue was found to stabilize the thiolate anion of GSH and enhance its nucleophilicity, while the Y residue was important in regulating catalytic function. The GSTU25-GSSG structure also revealed that the terminal carboxylate group of the GSH-1 $\gamma$-glutamyl moiety formed an interaction at $2.6 \AA$ with the nitrogen atom of the guanidinium group of R111, and that the glycine moiety of GSH-1 protruded toward the GSTU25 $\alpha 4$ chain. The GSH-2 molecule, at the carboxylate terminal of the glycine moiety, formed an interaction with the oxygen atom of K40 at a distance of $2.7 \AA$ with the $\gamma$-glutamyl moiety located in between the helices $\alpha 1$ and $\alpha 3$.

A Clostridium oremlandii glutaredoxin (CoGRX2) with two GSSG molecules per dimer has been reported (Lee et al., 2014) and exhibits significant similarity with GSTU25 at the core of the thioredoxin fold where four $\beta$-strands and $\alpha$-helices, can be observed (Figure 3A). In GSTU25, a serine interacts with the

TABLE 3 | Amino acid substitutions in the GSTU24 and GSTU25 mutants.

\begin{tabular}{ccc}
\hline Enzyme & Mutation identifier & Substitution \\
\hline GSTU24 & A & A12P \\
& B & N107Y \\
C & A115G \\
D & I208V \\
& E & R211L \\
GSTU25 & F & P12A \\
& G & Y107N \\
& H & G115A \\
& I & V209I \\
& J & L212R
\end{tabular}


GSSG molecule (Figure 3B), whereas in CoGRX2, cysteine acts as the GSH thiol stabilizer.

\section{Identification of Target Amino Acids for Site-Directed Mutagenesis}

Seven key residues (F15, L37, K40, K53, E66, S67, R111) identified in the structure of GSTU25, and shown in Figure 2B with side-chain carbon atoms in blue, are all conserved in GSTU24. Comparisons with the structure of TaGSTU4-4 (Thom et al., 2002) and GmGSTU4-4 (Axarli et al., 2009) were used to highlight further amino acid residues in GSTU24 and GSTU25 that are likely to be involved in the formation of the hydrophobic $\mathrm{H}$-site and thus in the determination of substrate specificity (shown as orange-outlined boxes in Figure 4). Of the six residues known to be important for substrate specificity in Tau class GSTs (shown in blue boxes), the residue at position 107 ( $\mathrm{N}$ for GSTU24, Y for GSTU25) was the only one not identical between GSTU24 and GSTU25, and was thus targeted for mutagenesis. Subsequent homology modeling using the published structure of GmGSTU4-4 (Axarli et al., 2009), which shares high (>60\%) protein sequence identity with GSTU24 and GSTU25, identified four, further, non-identical residues, at positions 12, 115, 208 (209 for GSTU25) and 211 (212 for GSTU25), as shown in Figure 2B. The five amino acid residues targeted for reciprocal mutagenesis are listed in Table 3, marked as blue triangles in Figure 4, and highlighted with side-chain carbon atoms in gold for U25 in Figure 2B.

\section{Activity of GSTU24 and GSTU25 Mutants Toward TNT}

To determine the effects of the target mutations on the ability of the GST proteins to produce the three different TNT-conjugates, the mutated proteins were assayed using TNT as substrate. For GSTU24, mutation BCD significantly reduced $(p=0.003)$ overall levels of conjugates produced to $52 \%$ of the wild-type GSTU24, whereas mutations $\mathrm{AB}$ and $\mathrm{ABCDE}$ displayed significantly higher (82 and 163\%, respectively, $p<0.0001$ ) conjugating activity than the wild type GSTU24 (Figure 5A). Figure 5B shows that all five mutants were able to produce conjugate 1 , which was not detectable from wild type GSTU24 under these conditions. The mutant ABCDE was distinct from the others tested as it displayed the highest overall conjugating activity of the five U25-derived mutants. This ABCDE mutant was also able to produce significantly higher $(p<0.0001)$ amounts of conjugate 3, GDNT, than GSTU24, or the other four mutants. Moreover, $\mathrm{ABCDE}$ produced all three conjugates in almost equimolar concentrations.

For the GSTU25, mutations, FG and G significantly reduced ( $p<0.0001$ ) overall levels of conjugates produced to 31 and $24 \%$, respectively, of the wild-type GSTU25. Compared to GSTU25,

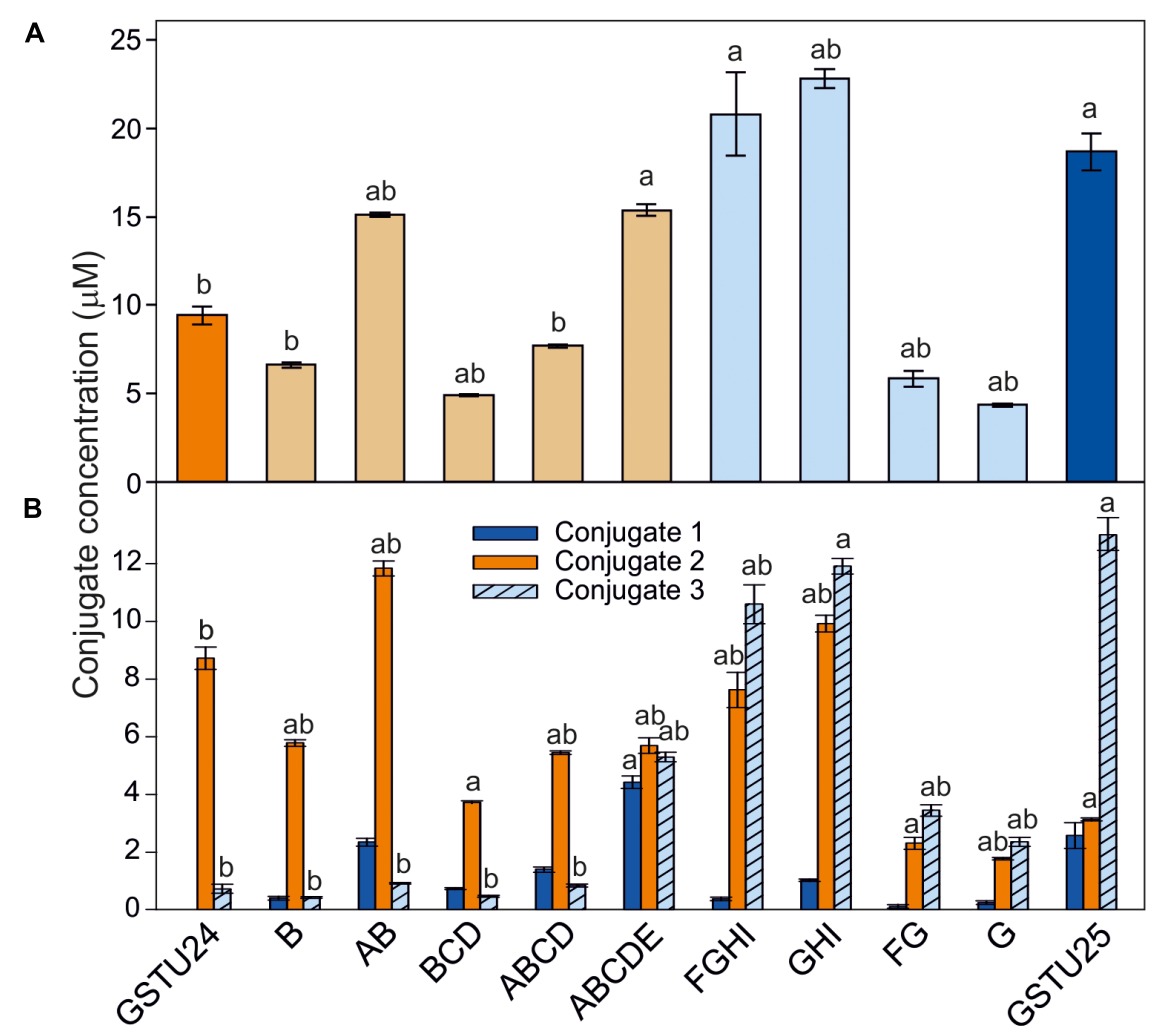

FIGURE 5 | TNT-conjugate profiles from GSTs. (A) Total conjugates and (B) conjugate profiles produced by AtGSTU24, AtGSTU25, and mutants. Conjugate 3 = 2-glutathionyl-4,6-dinitrotoluene (GDNT). Results are means of three replicates $\pm \mathrm{SE}$, a, significantly different from AtGSTU24, b, significantly different from AtGSTU25. 
these FG and $G$ mutants also yielded significantly reduced $(p<0.0001)$ overall levels of GDNT, while levels of conjugate 2 were not significantly affected for $\mathrm{G}$ (Figure 5B). Mutant GHI produced significantly more overall conjugates $(p=0.007)$ when compared to wild-type GSTU25, with both GHI and FGHI also producing significantly more conjugate $2(p<0.0001)$.

\section{Probing the GSTU24 and GSTU25 Mutants for Conformational Changes}

To identify any conformational changes in protein structure resulting from the presence of the mutated residues, the mutants were probed with 1-anilino-8-naphthalene-sulfonate (ANS) and the spectra measured. Both GSTU24 and GSTU25 shared a similar structure in the hydrophobic site (Figure 6A), with only the ABCDE mutant generating a significantly different fluorescence spectrum, indicative of a change in conformation

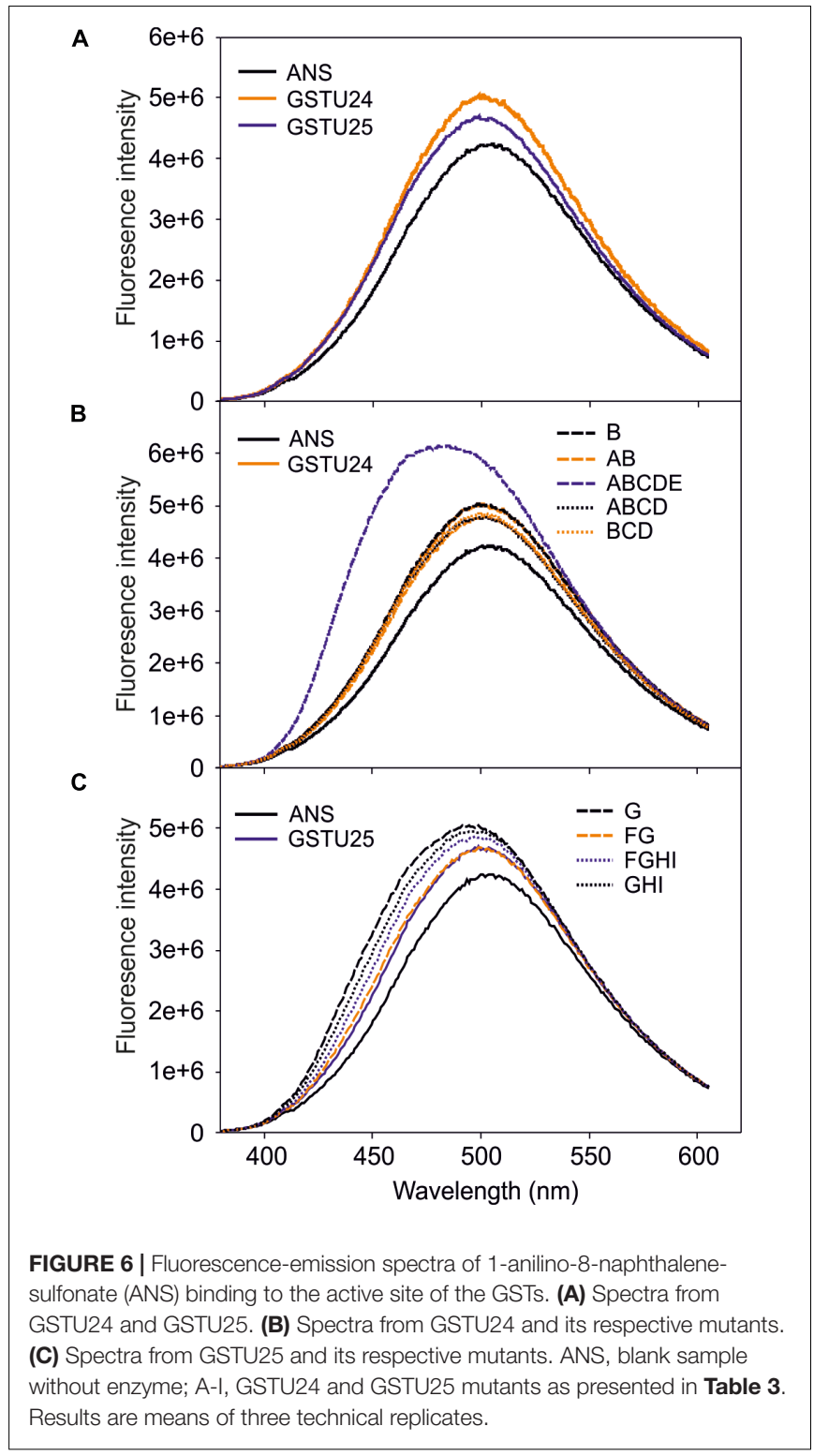

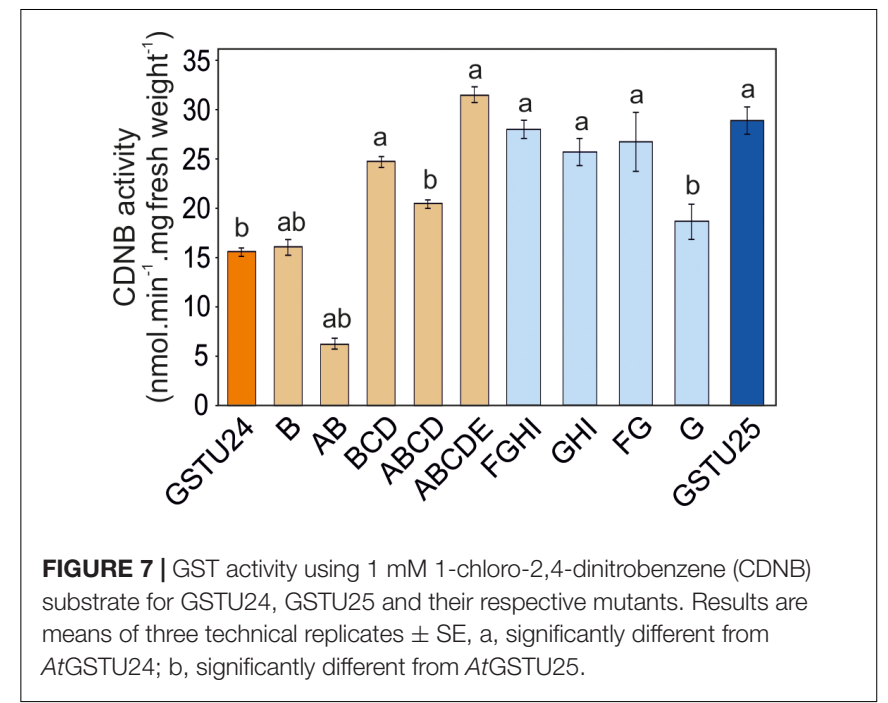

(Figure 6B). The fluorescence spectra of the different GSTU25 mutants, varied slightly to one another, but none of them suggested a significant conformational change had occurred (Figure 6C).

\section{Activity of GSTU24, GSTU25, and Mutants Toward CDNB}

The activity of the mutants was measured using CDNB as a substrate. The results in Figure 7 show that all the mutants exhibited changes in activity that were significantly different to either, or both of the wild type GSTs. Given that the mutant $\mathrm{ABCDE}$ was distinct in displaying the highest overall conjugating activity, and producing significantly higher amounts of the desired target, GDNT, kinetic analysis was performed using CDNB substrate (Figure 8 and Table 4). While GSTU24 and GSTU25 exhibited similar $\mathrm{V}_{\max }$ values, the $K_{\mathrm{m}}$ for GSTU24 was 45-fold higher than for GSTU25. In agreement with our reported conjugate profiles, the GSTU24 ABCDE mutant also displayed a reduced, GSTU25-like, $K_{\mathrm{m}}$ value.

\section{DISCUSSION}

The aim of this study was to identify the amino acids within GSTU25 involved in the formation of GDNT. To achieve this, the structure of GSTU25 was first determined. The structure, along with comparisons with the known Tau class GST structures TaGSTU4-4 (Thom et al., 2002) and GmGSTU4-4 (Axarli et al., 2009); and amino acid sequence of the closely related GSTU24, were used to highlight the amino acid residues in GSTU25 most likely to be involved.

\section{Crystal Structure of GSTU25}

The electron density map for GSTU25 revealed unambiguously one GSSG per subunit. Within the GSTU25-GSSG structure, the GSH-1 moiety is stabilized by an arginine side chain (R111) while the GSH-2 moiety is located at a well-documented GSH binding site (Axarli et al., 2009, 2016; Skopelitou et al., 2015). The binding 


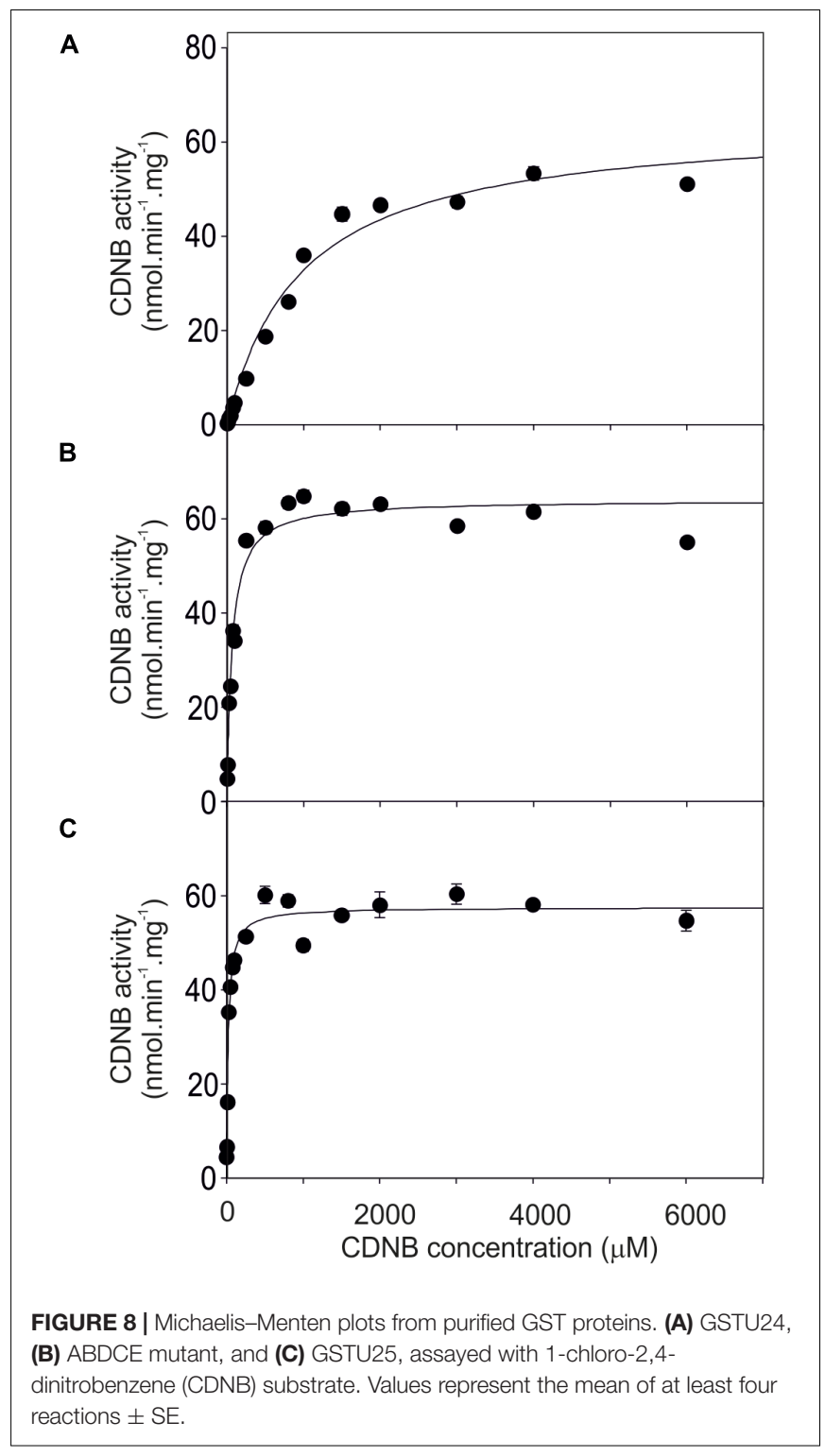

of GSTs to GSSG has been reported in the wood fungus, Phanerochaete chrysosporium PcUre2pA (Roret et al., 2015), and E. coli homologs EcYghU and EcYfcG. These bacterial and fungal GSTs have GSH transferase activity and are distantly related to glutaredoxins, redox enzymes that reduce disulfide bonds using glutathione (GSH) as an electron donor (Stourman et al., 2011). As shown in Figure 3, U25 shares significant similarity CoGRX2. In yeast (Saccharomyces cerevisiae), glutaredoxins ScGRX1 and ScGRX2 display GST-like activities, catalyzing the conjugation of

TABLE 4 | Enzyme kinetics for Figure 8, assayed using CDNB substrate.

\begin{tabular}{lccc}
\hline Enzyme & $\boldsymbol{K}_{\mathbf{m}}(\boldsymbol{\mu} \mathbf{M})$ & $\mathbf{V}_{\mathbf{m a x}}\left(\mathbf{n m o l e ~} \mathbf{~} \mathbf{n i n}^{-\mathbf{1}} \mathbf{~ m g}^{-\mathbf{1}}\right)$ & $\boldsymbol{R}^{\mathbf{2}}$ \\
\hline GSTU24 & $972 \pm 72.9$ & $64.7 \pm 1.6$ & 0.98 \\
ABCDE & $64.8 \pm 4.3$ & $64.0 \pm 0.8$ & 0.96 \\
GSTU25 & $21.5 \pm 1.9$ & $57.6 \pm 0.7$ & 0.95
\end{tabular}

CDNB to GSH (Collinson and Grant, 2003). As a multifunctional enzyme, exhibiting glutaredoxin, GPOX, and GST activities, GSTU25 would be well-suited to detoxify a wide range of the xenobiotics and oxidants present in diverse stress conditions.

\section{Residues Important to TNT-Conjugation Activity}

Both GSTU24 and GSTU25 contain a serine residue (S13) in the active site at a position that allows it to stabilize the thiolate anion of glutathione. This is in agreement with structures of GSTs from Theta and Phi classes that are known to have GSH conjugating activity (Thom et al., 2001) and is replaced by cysteine for Lambda and DHAR GSTs (Dixon et al., 2002). The effects of the mutations on the activity toward TNT showed that Y107 in GSTU25 is important for conjugate specificity. GSTU24 does not produce conjugate 1 under the conditions tested; however, the N107Y mutation confers the ability to produce albeit small (6\%) amounts of this conjugate. The data presented here also indicate that high activity of toward TNT requires both Y107 and P12. At the binding site of GSTU25, the GSSG subunits are located in a binding pocket surrounded by polar, non-polar and charged amino acids; a well-characterized active site for GSTs (Brock et al., 2013). In GmGSTU4-4, the same S and Y residues of GmGSTU44 are present in this binding pocket. When in complex with $\underline{S}$ - $(p$-nitrobenzyl)-glutathione, the S residue stabilizes the thiolate anion of GSH and enhances its nucleophilicity, while the Y residue is important in regulating catalytic function (Axarli et al., 2009). In GST25, L212 could also contribute to the production of GDNT; in GmGSTU4-4, the close proximity of this residue to the nitro group of 4-nitrobenzyl (Axarli et al., 2009) could orientate TNT in the active site.

The five consecutive mutations present in GSTU25 ABCDE were predicted to engineer the near-complete active site of GSTU25 into GSTU24. The resulting conjugate profile and activity of ABCDE were similar to GSTU25 in that all three conjugates were produced, and at levels of overall conjugating activity similar to those of GSTU24. Furthermore, the $K_{\mathrm{m}}$ value of ABCDE was more in-line with that of GSTU25. Nonetheless, the fluorescence emission spectrum of ABCDE was significantly different from both GSTU24 and GSTU25, indicating a conformational change in the hydrophobic site, and TNT was not crystalized within the GSTU25 structure. Although TNT and reduced GSH were supplied during the crystallization process, incorporation of TNT into the active site was likely to have been hindered by the low aqueous solubility of TNT. Using synthesized, and more soluble, GDNT, in the absence of GSH, during the crystallization process could perhaps yield more information about the residues involved during the formation of this conjugate.

In summary, we have solved the structure for GSTU25, and identified key residues involved in the formation of 2-GDNT. Substitution of a nitro group for sulfur in 2-GDNT could render the aromatic ring more susceptible to subsequent degradation, and endogenous degradative pathways may already exist in planta. Alternatively, both bacteria and fungi are able to mineralize DNT (Serrano-González et al., 2018), and may 
have activity toward 2-GDNT. These fundamental studies will contribute toward the development of plant-based remediation strategies to degrade TNT, a toxic environmental pollutant.

\section{AUTHOR CONTRIBUTIONS}

KT, LA, and MD carried out the experiments. ER took the lead in writing the manuscript. All authors conceived and planned the experiments, provided critical feedback, helped shape the research, performed the analysis, and wrote the manuscript.

\section{REFERENCES}

Amaral, H. I., Fernandes, J., Berg, M., Schwarzenbach, R. P., and Kipfer, R. (2009). Assessing TNT and DNT groundwater contamination by compound-specific isotope analysis and $3 \mathrm{H}-3 \mathrm{He}$ groundwater dating: a case study in Portugal. Chemosphere 77, 805-812. doi: 10.1016/j.chemosphere.2009.08.011

Axarli, I., Dhavala, P., Papageorgiou, A. C., and Labrou, N. E. (2009). Crystallographic and functional characterization of the fluorodifen-inducible glutathione transferase from Glycine max reveals an active site topography suited for diphenylether herbicides and a novel L-site. J. Mol. Biol. 385, 9841002. doi: 10.1016/j.jmb.2008.10.084

Axarli, I., Muleta, A. W., Vlachakis, D., Kossida, S., Kotzia, G., Maltezos, A., et al. (2016). Directed evolution of Tau class glutathione transferases reveals a site that regulates catalytic efficiency and masks co-operativity. Biochem. J. 473, 559-570. doi: 10.1042/bj20150930

Brock, J., Board, P. G., and Oakley, A. J. (2013). Structural insights into omega-class glutathione transferases: a snapshot of enzyme reduction and identification of a non-catalytic ligandin site. PLoS One 8:e60324. doi: 10.1371/journal.pone. 0060324

Clark, B., and Boopathy, R. (2007). Evaluation of bioremediation methods for the treatment of soil contaminated with explosives in Louisiana Army Ammunition Plant, Minden, Louisiana. J. Hazard. Mater. 143, 643-648. doi: 10.1016/j. jhazmat.2007.01.034

Collinson, E. J., and Grant, C. M. (2003). Role of yeast glutaredoxins as glutathione S-transferases. J. Biol. Chem. 278, 22492-22497. doi: 10.1074/jbc.M301387200

Colville, L., and Smirnoff, N. (2008). Antioxidant status, peroxidase activity, and PR protein transcript levels in ascorbate-deficient Arabidopsis thaliana vtc mutants. J. Exp. Bot. 59, 3857-3868. doi: 10.1093/jxb/ern229

Dixon, D., Davis, B., and Edwards, R. (2002). Functional divergence in the glutathione transferase superfamily in plants - Identification of two classes with putative functions in redox homeostasis in Arabidopsis thaliana. J. Biol. Chem. 277, 30859-30869. doi: 10.1074/jbc.M202919200

Dixon, D., and Edwards, R. (2009). Selective binding of glutathione conjugates of fatty acid derivatives by plant glutathione transferases. J. Biol. Chem. 284, 21249-21256. doi: 10.1074/jbc.M109.020107

Dixon, D., Sellars, J., and Edwards, R. (2011). The Arabidopsis phi class glutathione transferase AtGSTF2: binding and regulation by biologically active heterocyclic ligands. Biochem. J. 438, 63-70. doi: 10.1042/BJ20101884

Dixon, D. P., and Edwards, R. (2010). Glutathione transferases. Arabidopsis Book 8:e0131. doi: 10.1199/tab.0131

Edwards, R., Dixon, D., Sies, H., and Packer, L. (2005). Plant glutathione transferases. Gluthione Transferases Gamma Glutamyl Transpeptidases 401, 169-186. doi: 10.1016/S0076-6879(05)01011-6

Emsley, P., and Cowtan, K. (2004). Coot: model-building tools for molecular graphics. Acta Crystallogr. D Biol.Crystallogr. 60, 2126-2132. doi: 10.1107/ S0907444904019158

Evans, P. (2006). Scaling and assessment of data quality. Acta Crystallogr. D Biol. Crystallogr. 62, 72-82. doi: 10.1107/s0907444905036693

French, C. E., Nicklin, S., and Bruce, N. C. (1998). Aerobic degradation of 2,4,6trinitrotoluene by Enterobacter cloacae $\mathrm{PB} 2$ and by pentaerythritol tetranitrate reductase. Appl. Environ. Microbiol. 64, 2864-2868.

Gandia-Herrero, F., Lorenz, A., Larson, T., Graham, I. A., Bowles, D. J., Rylott, E. L., et al. (2008). Detoxification of the explosive 2,4,6-trinitrotoluene in

\section{ACKNOWLEDGMENTS}

This work was funded by the United Kingdom Biotechnology and Biological Sciences Research Council (BB/P005713/1), and the Strategic Environmental Research and Development Program (ER-1498) and Environmental Security Technology Certification Program (ER-201436) of the United States Department of Defense. KT acknowledges funding from a Burgess studentship and LA funding for a studentship from a Majlis Amanah Rakyat (MARA), Malaysia. This study used the University of York Technology Facilities.

Arabidopsis: discovery of bifunctional O- and C-glucosyltransferases. Plant J. 56, 963-974. doi: 10.1111/j.1365-313X.2008.03653.x

Gunning, V., Tzafestas, K., Sparrow, H., Johnston, E. J., Brentnall, A. S., Potts, J. R., et al. (2014). Arabidopsis glutathione transferases U24 and U25 exhibit a range of detoxification activities with the environmental pollutant and explosive, 2,4,6-Trinitrotoluene. Plant Physiol. 165, 854-865. doi: 10.1104/pp.114.237180

Holm, L., and Rosenstrom, P. (2010). Dali server: conservation mapping in 3D. Nucleic Acids Res. 38, W545-W549. doi: 10.1093/nar/gkq366

Jenkins, T. F., Hewitt, A. D., Grant, C. L., Thiboutot, S., Ampleman, G., Walsh, M. E., et al. (2006). Identity and distribution of residues of energetic compounds at army live-fire training ranges. Chemosphere 63, 1280-1290. doi: 10.1016/j. chemosphere.2005.09.066

Johnson, G. R., Jain, R. K., and Spain, J. C. (2002). Origins of the 2,4-dinitrotoluene pathway. J. Bacteriol. 184, 4219-4232. doi: 10.1128/JB.184.15.4219-4232.2002

Kabsch, W. (2010). XDS. Acta Crystallogr. D Biol. Crystallogr. 66, 125-132. doi: 10.1107/s0907444909047337

Labrou, N. E., Papageorgiou, A. C., Pavli, O., and Flemetakis, E. (2015). Plant GSTome: structure and functional role in xenome network and plant stress response. Curr. Opin. Biotechnol. 32, 186-194. doi: 10.1016/j.copbio.2014.12. 024

Lee, E. H., Kim, H. Y., and Hwang, K. Y. (2014). The GSH- and GSSGbound structures of glutaredoxin from Clostridium oremlandii. Arch. Biochem. Biophys. 564, 20-25. doi: 10.1016/j.abb.2014.09.001

Lewis, T. A., Newcombe, D. A., and Crawford, R. L. (2004). Bioremediation of soils contaminated with explosives. J.Environ.Manage. 70, 291-307. doi: 10.1016/j. jenvman.2003.12.005

Mezzari, M. P., Walters, K., Jelinkova, M., Shih, M. C., Just, C. L., and Schnoor, J. L. (2005). Gene expression and microscopic analysis of arabidopsis exposed to chloroacetanilide herbicides and explosive compounds. A Phytoremediation Approach. Plant Physiol. 138, 858-869. doi: 10.1104/pp.104.056168

Murshudov, G. N., Skubák, P., Lebedev, A. A., Pannu, N. S., Steiner, R. A., Nicholls, R. A., et al. (2011). REFMAC5 for the refinement of macromolecular crystal structures. Acta Crystallogr. D Biol. Crystallogr. 67, 355-367. doi: 10.1107/ S0907444911001314

Murshudov, G. N., Vagin, A. A., and Dodson, E. J. (1997). Refinement of macromolecular structures by the maximum-likelihood method. Acta Crystallogr. D Biol. Crystallogr. 53, 240-255. doi: 10.1107/S0907444996012255

Nishino, S. F., Paoli, G. C., and Spain, J. C. (2000). Aerobic degradation of dinitrotoluenes and pathway for bacterial degradation of 2,6-dinitrotoluene. Appl. Environ. Microbiol. 66, 2139-2147. doi: 10.1128/AEM.66.5.2139-2147. 2000

Qasim, M., Gorb, L., Magers, D., Honea, P., Leszczynski, J., Moore, B., et al. (2009). Structure and reactivity of TNT and related species: application of spectroscopic approaches and quantum-chemical approximations toward understanding transformation mechanisms. J. Hazard. Mater. 167, 154-163. doi: 10.1016/j. ihazmat.2008.12.105

Roret, T., Thuillier, A., Favier, F., Gelhaye, E., Didierjean, C., and MorelRouhier, M. (2015). Evolutionary divergence of Ure2pA glutathione transferases in wood degrading fungi. Fungal Genet. Biol. 83, 103-112. doi: 10.1016/j.fgb.2015.09.002

Rylott, E. L., Lorenz, A., and Bruce, N. C. (2011). Biodegradation and biotransformation of explosives. Curr. Opin. Biotechnol. 22, 434-440. doi: 10. 1016/j.copbio.2010.10.014 
Serrano-González, M. Y., Chandra, R., Castillo-Zacarias, C., Robledo-Padilla, F., Rostro-Alanis, M. D. J., and Parra-Saldivar, R. (2018). Biotransformation and degradation of 2,4,6-trinitrotoluene by microbial metabolism and their interaction. Defence Technol. 14, 151-164. doi: 10.1016/j.dt.2018.01.004

Sievers, F., Wilm, A., Dineen, D., Gibson, T. J., Karplus, K., Li, W., et al. (2011). Fast, scalable generation of high-quality protein multiple sequence alignments using Clustal Omega. Mol. Syst. Biol. 7:539. doi: 10.1038/msb.2011.75

Skopelitou, K., Muleta, A. W., Papageorgiou, A. C., Chronopoulou, E., and Labrou, N. E. (2015). Catalytic features and crystal structure of a tau class glutathione transferase from Glycine max specifically upregulated in response to soybean mosaic virus infections. Biochim. Biophys. Acta 1854, 166-177. doi: 10.1016/j. bbapap.2014.11.008

Smith, A. P., Nourizadeh, S. D., Peer, W. A., Xu, J., Bandyopadhyay, A., Murphy, A. S., et al. (2003). Arabidopsis AtGSTF2 is regulated by ethylene and auxin, and encodes a glutathione S-transferase that interacts with flavonoids. Plant J. 36, 433-442. doi: 10.1046/j.1365-313X.2003.01890.x

Spain, J. C., Hughes, J. B., and Knackmuss, H. J. (2000). Biodegradation of Nitroaromatic Compounds and Explosives. Boca Raton, FL: Lewis.

Stourman, N. V., Branch, M. C., Schaab, M. R., Harp, J. M., Ladner, J. E., and Armstrong, R. N. (2011). Structure and function of YghU, a nu-class glutathione transferase related to YfcG from Escherichia coli. Biochemistry 50, 1274-1281. doi: 10.1021/bi101861a

Talmage, S. S., Opresko, D. M., Maxwell, C. J., Welsh, C. J., Cretella, F. M., Reno, P. H., et al. (1999). Nitroaromatic munition compounds: environmental effects and screening values. Rev. Environ. Contam. Toxicol. 161, 1-156. doi: 10.1007/ 978-1-4757-6427-7_1

Thom, R., Cummins, I., Dixon, D. P., Edwards, R., Cole, D. J., and Lapthorn, A. J. (2002). Structure of a tau class glutathione S-transferase from wheat active in herbicide detoxification. Biochemistry 41, 7008-7020. doi: 10.1021/bi015964x

Thom, R., Dixon, D., Edwards, R., Cole, D., and Lapthorn, A. (2001). The structure of a zeta class glutathione S-transferase from Arabidopsis thaliana: characterisation of a GST with novel active-site architecture and a putative role in tyrosine catabolism. J. Mol. Biol. 308, 949-962. doi: 10.1006/jmbi.2001.4638
United States General Accounting Office (2004). Department of Defense Operational Ranges, More Reliable Cleanup Cost Estimates and a Proactive Approach to Identifying Contamination are Needed. Available at: http://www. gao.gov/new.items/d04601.pdf

Vagin, A., and Teplyakov, A. (1997). MOLREP: an automated program for molecular replacement. J. Appl. Crystallogr. 30, 1022-1025. doi: 10.1107/ S0021889897006766

Valli, K., Brock, B. J., Joshi, D. K., and Gold, M. H. (1992). Degradation of 2,4dinitrotoluene by the lignin-degrading fungus Phanerochaete chrysosporium. Appl. Environ. Microbiol. 58, 221-228.

Winter, G. (2010). xia2: an expert system for macromolecular crystallography data reduction. J. Appl. Crystallogr. 43, 186-190. doi: 10.1107/S002188980904 5701

Yang, X., Sun, W., Liu, J.-P., Liu, Y.-J., and Zeng, Q.-Y. (2009). Biochemical and physiological characterization of a tau class glutathione transferase from rice (Oryza sativa). Plant Physiol. Biochem. 47, 1061-1068. doi: 10.1016/j.plaphy. 2009.07.003

Zheng, W., Lichwa, J., D'alessio, M., and Ray, C. (2009). Fate and transport of TNT, RDX, and HMX in streambed sediments: implications for riverbank filtration. Chemosphere 76, 1167-1177. doi: 10.1016/j.chemosphere.2009. 06.043

Conflict of Interest Statement: The authors declare that the research was conducted in the absence of any commercial or financial relationships that could be construed as a potential conflict of interest.

Copyright (C) 2018 Tzafestas, Ahmad, Dani, Grogan, Rylott and Bruce. This is an open-access article distributed under the terms of the Creative Commons Attribution License (CC BY). The use, distribution or reproduction in other forums is permitted, provided the original author(s) and the copyright owner(s) are credited and that the original publication in this journal is cited, in accordance with accepted academic practice. No use, distribution or reproduction is permitted which does not comply with these terms. 